Check for updates

Cite this: RSC Adv., 2017, 7, 41399

Received 15th July 2017

Accepted 19th August 2017

DOI: 10.1039/c7ra07801c

rsc.li/rsc-advances

\section{Synthesis of hollow anatase nanospheres with excellent adsorption and photocatalytic performances}

\author{
Dongjun Chen, ${ }^{\text {a }}$ Fangping Ma, ${ }^{\mathrm{b}}$ Bo Lei, ${ }^{\mathrm{b}}$ Wei Qiu, ${ }^{\mathrm{b}}$ Xinbai Jiang, ${ }^{\mathrm{a}}$ Hongxia Yu, (D) *a \\ Huiping $\mathrm{Bi}^{a}$ Yong Yang ${ }^{a}$ and Jinyou Shen*a
}

\begin{abstract}
Hollow anatase nanospheres have been synthesized. The silica template makes anatase more stable and its subsequent removal results in the formation of hollow structures, which significantly improves its surface area and active sites. The as-synthesized hollow anatase nanospheres show excellent adsorption and photocatalytic performance to solid anatase nanospheres and P25.
\end{abstract}

$\mathrm{TiO}_{2}$ has been widely used as a photocatalyst in applications such as water treatment and purification. ${ }^{1}$ However, the low utilization efficiency of solar energy and easy recombination of photogenerated electrons and holes limited its practical applications. ${ }^{2}$ Therefore, much effort has been devoted to enhancing the photocatalytic performance of $\mathrm{TiO}_{2}$. It has been shown that crystal phases and crystallinity are playing important roles in increasing photocatalytic efficiency., ${ }^{3,4}$ Anatase is considered as the most active phase because of its high reduction potential and lower recombination rate of electron-hole pairs. ${ }^{5}$ Although high crystallinity is preferred to enhance the photocatalytic activity, anatase is thermodynamically metastable due to its tendency to transform to rutile at $600{ }^{\circ} \mathrm{C}$. Therefore, the stability of anatase deserved to be improved. Moreover, morphology also plays an important role in promoting the photocatalytic efficiency. It is well recognized that hollow structures are beneficial for facilitating the ionic transport and supplying more redox reaction sites. Synthesis of hollow $\mathrm{TiO}_{2}$ has been extensively reported, mainly through Ostwald ripening, ${ }^{6}$ Kirkendall effect ${ }^{7}$ or template methods. ${ }^{8}$ It is thus of great importance to prepare $\mathrm{TiO}_{2}$ with special crystalline phase and hollow structures. There are also some reports of the preparation of $\mathrm{TiO}_{2}$ hollow spheres. ${ }^{9,10}$ However, there are still great challenges in preparing $\mathrm{TiO}_{2}$ hollow spheres which possesses excellent adsorption and photocatalytic performances.

In this paper, we further explore the synthesis of hollow anatase nanospheres with excellent adsorption and photocatalytic properties. First, $\mathrm{SiO}_{2}$ was prepared through modified Stöber method and coated with $\mathrm{TiO}_{2}$ through the hydrolysis of tetrabutyl titanate (ТВОT). Then, the as-prepared $\mathrm{SiO}_{2} @ \mathrm{TiO}_{2}$

ajiangsu Key Laboratory for Chemical Pollution Control and Resource Reuse, School of Environmental and Biological Engineering, Nanjing University of Science and Technology, Nanjing 210094, Jiangsu Province, China. E-mail: yhx19860101@163. com; shenjinyou@mail.njust.edu.cn

${ }^{b}$ Chuannan Machinery Factory, China Aerospace Science and Technology Corporation, Luzhou 646000, Sichuan Province, China
(ST) was calcinated at different temperatures. Finally, $\mathrm{SiO}_{2}$ was etched away by using $\mathrm{NaOH}$ solution to obtain hollow anatase nanospheres. The adsorption and photocatalytic properties of as-synthesized samples for removing and photodegradation Methylene Blue (MB) were investigated.

The hollow anatase nanospheres were synthesized using hard template method, as indicated in Fig. 1a. $\mathrm{SiO}_{2}$ was prepared through modified Stöber method ${ }^{\mathbf{1 1}}$ and dispersed into anhydrous ethanol for further use. $0.1 \mathrm{~g}$ of hydroxypropyl cellulose was dissolved into a mixture of $0.12 \mathrm{~mL}$ of DI water and $25 \mathrm{~mL}$ of anhydrous ethanol, and $0.1 \mathrm{~g} \mathrm{SiO}_{2}$ in $0.5 \mathrm{~mL}$ anhydrous ethanol was injected. $1 \mathrm{~mL}$ of TBOT in $4.5 \mathrm{~mL}$ of



Fig. 1 (a) Schematic illustration for the synthesis of hollow anatase nanospheres. TEM images of (b) $\mathrm{SiO}_{2}$ nanoparticles, (c) ST nanoparticles and (d) hollow anatase nanospheres. 
anhydrous ethanol was dripped into the mixture and stirred to homogeneity. The system was kept at $85{ }^{\circ} \mathrm{C}$ for 90 min under refluxing condition. The product was collected by centrifugation and washed with anhydrous ethanol three times. After drying, the product was calcined at the desired temperatures for $2 \mathrm{~h}$ under air condition. Finally, $\mathrm{SiO}_{2}$ was etched away by using $\mathrm{NaOH}$ solution $(2 \mathrm{M}, 30 \mathrm{~mL})$ to obtained hollow anatase nanospheres, labeled as "ST- $x$-E", $x$ represent temperature.

Fig. 1b shows the TEM image of $\mathrm{SiO}_{2}$ nanospheres with smooth surface and diameter of $\sim 350 \mathrm{~nm}$. After coating with a layer of titania, the surface of $\mathrm{SiO}_{2}$ became rough and the diameter increased to $\sim 390 \mathrm{~nm}$ (Fig. 1c). ST were then calcined at aimed temperature to convert titania into anatase. Finally, $\mathrm{NaOH}$ was used to remove the silica-templates and the final product of hollow anatase nanospheres with the thickness of $20 \mathrm{~nm}$ was synthesized (Fig. 1d).

XRD has been used to study the crystalline phase of ST nanoparticles. As shown in Fig. 2a, all the samples transformed to single anatase phase (JCPDS no. 21-1272) and showed obvious peak at $2 \theta=25.3,37.8,48.0,53.9,55.0,62.7^{\circ}$ which are corresponding to the (101), (004), (200), (105), (211), and (204) planes of crystalline structure of anatase. With increasing the temperature, the intensities increase while slight increase is observed when the temperature increases from $800{ }^{\circ} \mathrm{C}$ to $900{ }^{\circ} \mathrm{C}$. It is well known that the anatase-rutile transformation usually happens in the temperature range of $600-700{ }^{\circ} \mathrm{C} .{ }^{\mathbf{1 2}}$ However, the crystalline phase of the sample remains pure anatase when ST particles were calcined at $700-900{ }^{\circ} \mathrm{C}$. It might be explained by the inhibition of $\mathrm{TiO}_{2}$ crystal grain-growth by the existence of silicate species. ${ }^{\mathbf{1 0}}$

The surface functionality of ST and ST-500 E was studied by FT-IR spectroscopy (Fig. 2b). The broad and strong band observed at $1100 \mathrm{~cm}^{-1}$ could be assigned to the antisymmetric stretching vibration of $\mathrm{Si}-\mathrm{O}-\mathrm{Si}$. Furthermore, the band at $950 \mathrm{~cm}^{-1}$ is due to the $\mathrm{Si}-\mathrm{O}-\mathrm{Ti}$ bond vibration, suggesting that $\mathrm{TiO}_{2}$ was connected with $\mathrm{SiO}_{2}$ by chemical bond rather than physical effects. For ST-500 E sample, these peaks are absent because $\mathrm{SiO}_{2}$ was etched by $\mathrm{NaOH}$ thoroughly. Ti-O-Ti stretching show transmittance peaks around $590 \mathrm{~cm}^{-1}$, both samples of ST and ST-500 E show obvious peaks in those position, it reflects that calcination and etching will not change the component of $\mathrm{TiO}_{2}$. In addition, the IR peaks at $1640 \mathrm{~cm}^{-1}$ and $3400 \mathrm{~cm}^{-1}$ can be ascribed to surface hydroxyl and adsorbed water molecules, which make the samples more accessible to water.

$\mathrm{N}_{2}$ adsorption-desorption isotherms and the corresponding pore size distribution plots of sample ST-500 E were shown in Fig. 2c and d respectively. The isotherm presents a typical IVtype hysteresis loop, indicating the presence of mesopores. The pore size distribution, which was calculated from the desorption branch of the nitrogen isotherm by the BarrettJoyner-Halenda (BJH) method, shows one groups of mesopores with pore diameters $\sim 3.7 \mathrm{~nm}$. The surface area calculated from the isotherm by the Brunauer-Emmett-Teller equation is $122.0 \mathrm{~m}^{2} \mathrm{~g}^{-1}$, which is higher than commercially used $\mathrm{TiO}_{2}$ photocatalyst P25 $\left(56.5 \mathrm{~m}^{2} \mathrm{~g}^{-1}\right)$, ST-600 E (109.1 $\left.\mathrm{m}^{2} \mathrm{~g}^{-1}\right)$, ST-700 E $\left(92.8 \mathrm{~m}^{2} \mathrm{~g}^{-1}\right)$, ST-800 E $\left(80.4 \mathrm{~m}^{2} \mathrm{~g}^{-1}\right)$ and ST-900 E $\left(64.6 \mathrm{~m}^{2} \mathrm{~g}^{-1}\right)$.

In order to characterize the photo-electrochemical property of the obtained hollow anatase nanospheres, chronoamperometry experiment was carried out in a standard threeelectrode cell. In the absence of UV light irradiation, almost all the samples showed a rather low current, i.e., $\sim 0 \mu \mathrm{A}$ (Fig. 3a). However, when the samples were irradiated by UV light, the photocurrent increased obviously because of the existence of photogenerated electrons. The intensity of photocurrent from
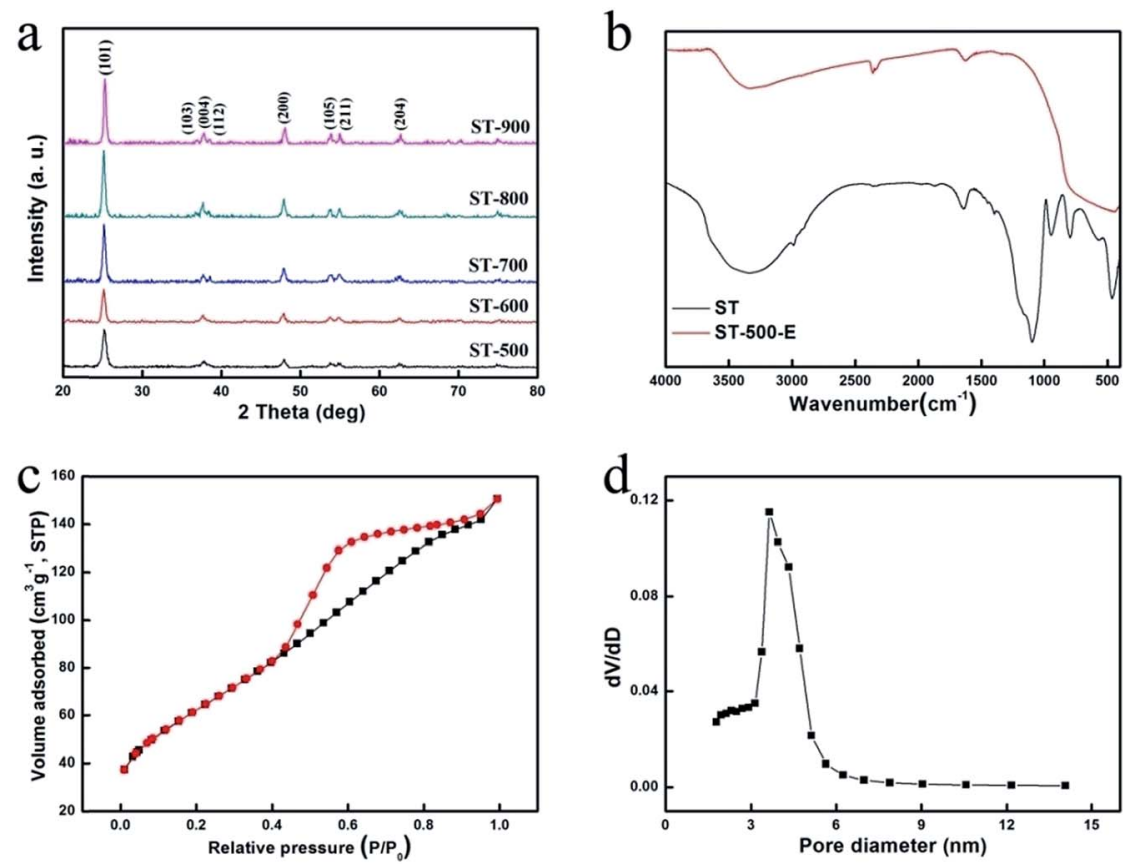

Fig. 2 (a) XRD patterns of ST calcined at different temperature, (b) FT-IR spectrum of ST and ST-500 E, (c) $\mathrm{N}_{2}$ adsorption-desorption isotherm and (d) pore size distribution of ST-500 E. 
the as-prepared hollow anatase nanoparticles was much higher than that of solid anatase nanoparticles which were calcined at $500{ }^{\circ} \mathrm{C}(\mathrm{T}-500)$, indicating the advantages of the hollow structure in separation efficiency of electrons and holes. With the increase of calcination temperature, the intensity of photocurrent increased due to the enhanced crystallinity of the samples. After several cycles of light illumination, photocurrent of ST-900 E decreased significantly, suggesting poor photocatalytic stability. However, photocurrent of ST-700 E and ST-500 E was quite stable, indicating excellent photocatalytic stability. Compared with ST-500 E, ST-900 E was characterized by high crystallinity and small surface area, indicating the existence of less high activated sites. Once deactivation of high activated sites would
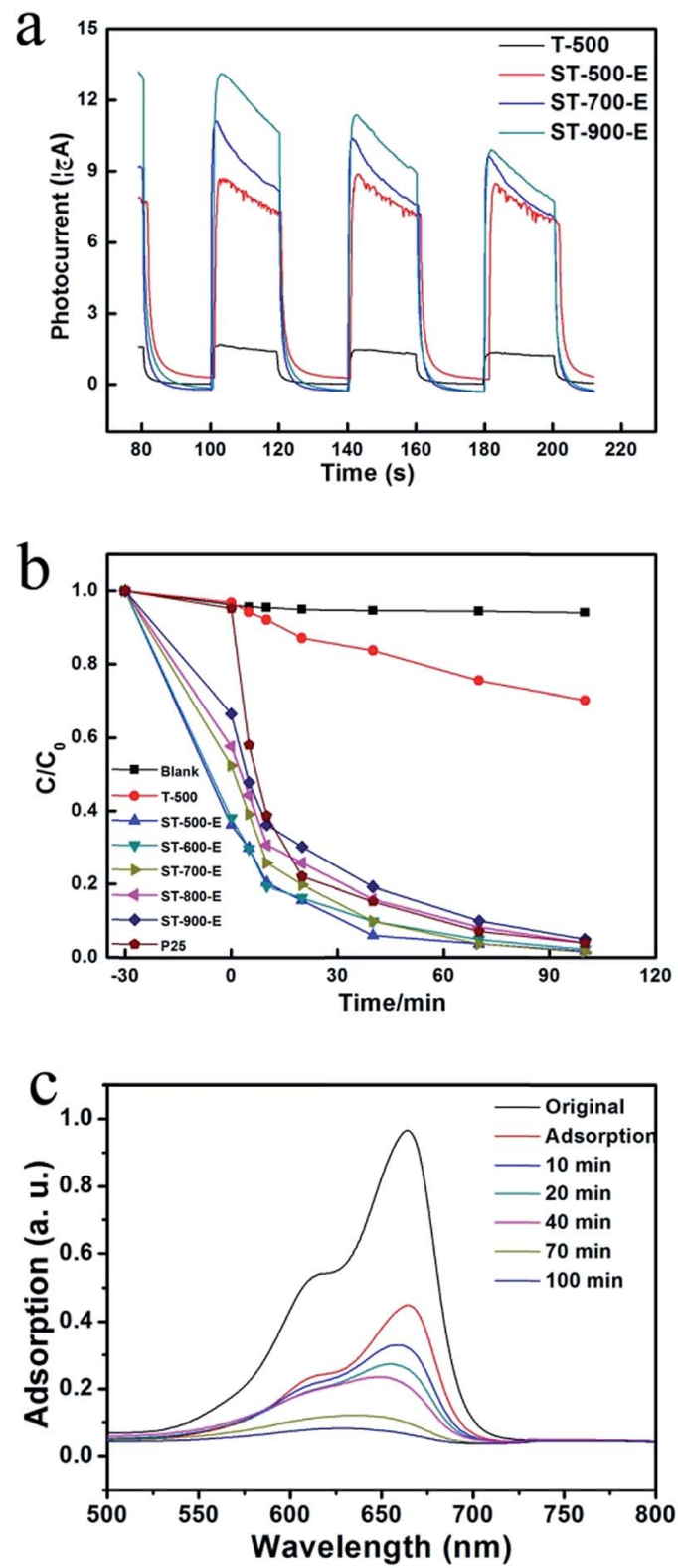

Fig. 3 (a) Chronoamperometry of hollow anatase nanospheres calcined at different temperatures, (b) photocatalytic degradation of $\mathrm{MB}$ with different $\mathrm{TiO}_{2}$ samples, and (c) the evolution of absorption spectra of MB solution at the presence of ST-500 E under UV light. occur, photogenerated current will decrease rapidly, resulting in bad photocatalytic stability. Therefore, the improved stability of ST-500 E is because of its large surface area.

Photocatalytic degradation of $20 \mathrm{mg} \mathrm{L}^{-1} \mathrm{MB}$ was carried out in $100.0 \mathrm{~mL}$ beakers containing $50.0 \mathrm{~mL} \mathrm{MB}$ solution. The asprepared hollow nanoparticles were added into the beakers as photocatalysts at the dosage of $15 \mathrm{mg}$. In order to evaluate the adsorption of $\mathrm{MB}$ onto the as-prepared photocatalysts, the aqueous slurry was stirred and sampled in dark condition for $30 \mathrm{~min}$ (graphically represented as $-30 \mathrm{~min}$ ) before UV illumination under $300 \mathrm{~W} \mathrm{Hg}$ lamp. The evolution of MB concentration during photocatalytic process using different photocatalysts was summarized in Fig. 3b. The adsorption capacity of hollow anatase nanospheres was better than P25 and T-500 because hollow structure offered higher surface area. The decrease of adsorption capacity for hollow anatase nanospheres obtained at higher temperature could be explained by the decrease of surface area with the increase of calcination temperature, which is consistent with the surface area measurements. The surface area of ST-900 E is higher than P25, but P25 shows better MB degradation than ST-900 E. There may be two reasons. One is that this may be attributed to the crystallinity of P25. The other is that the surface area of ST-900 E is just a little higher than P25, as a result of a narrow advantage in photocatalytic property. ST-500 E gave the highest photocatalytic and adsorption activity, implying large surface area, high crystallinity and special spatial structure. The evolution of absorption spectra was illustrated to further reveal the photocatalytic degradation process of MB at the presence of ST-500 E. As shown in Fig. 3c, the strong adsorption peak at $664 \mathrm{~nm}$ gradually diminished in intensity as the UV irradiation was prolonged, and disappeared after $100 \mathrm{~min}$, suggesting the complete photodegradation of $\mathrm{MB}$.

We have demonstrated the preparation of hollow anatase nanospheres with enhanced adsorption and photocatalytic activity. The synthesis involves the preparation of $\mathrm{SiO}_{2}$, coating of $\mathrm{TiO}_{2}$, thermal treatment, and the removal of $\mathrm{SiO}_{2} \cdot \mathrm{SiO}_{2}$ helps hollow anatase nanospheres maintain their crystalline phase during calcination at high temperatures, while the removal of $\mathrm{SiO}_{2}$ gives the hollow structures. Hollow anatase nanospheres with an optimal balance of high surface area and high crystallinity can be produced at a calcination temperature of $500{ }^{\circ} \mathrm{C}$, which ensures an enhanced adsorption and photocatalytic performance to solid anatase nanospheres and P25 in photodegradation of MB under UV light.

\section{Conflicts of interest}

There are no conflicts to declare.

\section{Acknowledgements}

This work was supported by the National Natural Science Foundation (51608269), Jiangsu Provincial Natural Science Foundation of China (BK20150778), Open Fund of Jiangsu Provincial Key Laboratory of biomass energy and materials (JSBEM201408), China Postdoctoral Science Foundation (1805) 
and Postdoctoral Science Foundation of Jiangsu Province (1402049B).

\section{Notes and references}

1 A. Fujishima, T. N. Rao and D. A. Tryk, J. Photochem. Photobiol., C, 2000, 1, 1.

2 A. L. Linsebigler, G. Lu and J. T. Yates, Chem. Rev., 1995, 95, 735.

3 J. B. Joo, Q. Zhang, M. Dahl, I. Lee, J. Goebl, F. Zaera and Y. Yin, Energy Environ. Sci., 2012, 5, 6321.

4 R. J. Dillon, J. B. Joo, F. Zaera, Y. Yin and C. J. Bardeen, Phys. Chem. Chem. Phys., 2013, 15, 1488.

5 J. B. Joo, Q. Zhang, I. Lee, M. Dahl, F. Zaera and Y. D. Yin, Adv. Funct. Mater., 2012, 22, 166.
6 J. Li and H. C. Zeng, J. Am. Chem. Soc., 2007, 129, 1583915847.

7 Y. Yin, R. M. Rioux, C. K. Erdonmez, S. Hughes, G. A. Somorjai and A. P. Alivisatos, Science, 2004, 304, 711.

8 L. Li, S. Zhou, E. Chen, R. Qiao, Y. Zhong, Y. Zhang and Z. Li, J. Mater. Chem. A, 2015, 3, 2234.

9 H. L. Shen, H. H. Hu, D. Y. Liang, H. L. Meng, P. G. Li, W. H. Tang and C. Cui, J. Alloys Compd., 2012, 542, 32.

10 H. Y. Liu, J. B. Joo, M. Dahl, L. S. Fu, Z. Z. Zeng and Y. D. Yin, Energy Environ. Sci., 2015, 8, 286.

11 Q. Zhang, T. Zhang, J. Ge and Y. Yin, Nano Lett., 2008, 8, 2867.

12 Y. Zhang, A. Weidenkaffa and A. Rellera, Mater. Lett., 2002, 54, 375. 